\title{
Case study on prepubescent vaginal bleeding
}

\author{
Charles Haddad ${ }^{1 *}$, Sofia Muenyi ${ }^{2}$ and Judella Haddad-Lacle ${ }^{3}$ \\ Department of Community Health and Family Medicine, University of Florida, USA
}

\begin{abstract}
Prepubertal vaginal bleeding is an abnormal symptom which elicits concern and prompt evaluation. The etiology can vary widely from vulvovaginitis to sexual abuse, foreign body and malignancy. A thorough history and physical examination by a healthcare provider is necessary for diagnosis. Pelvic ultrasound is usually the first modality of imaging for diagnosis, but X-rays and vaginoscopy may also aide in the diagnosis and treatment of prepubertal vaginal bleeding.
\end{abstract}

\section{Introduction}

Prepubertal vaginal bleeding is an abnormal symptom which elicits concern and prompt evaluation. The etiology can vary widely from vulvovaginitis to sexual abuse, foreign body and malignancy. Therefore, a thorough history and physical examination by a healthcare provider is necessary for diagnosis as even minor trauma can cause vaginal bleeding due to unestrogenized tissue. If the child is able to verbally communicate it is recommended to arrange interviewing them alone to corroborate reports and correlate exam findings. The number one concern for parents and providers when dealing with vaginal bleeding is sexual abuse. However, parents and providers are normally unaware of normative sexual behaviors for children at a pre-pubertal age. Between the ages of 2 to 5 , children may touch their own genitals at home or in public (25-44\%) or masturbate with their hand (10-16\%) [1]. Although when a child is nonverbal sometimes what may seem as possible masturbation may be a consequence of itching and irritation which leads to vaginal bleeding due to fragile prepubertal tissues [1]. In recent literature, genitourinary tract trauma is noted to be the most common cause of vaginal bleeding during hospital visits [2] while foreign bodies comprises approximately $4 \%$ of outpatient visits [3]. The most common foreign body is toilet paper but can range from lead pencils to safety pins [1]. If left undetected it can lead to complications and significant morbidity. However, literature on foreign bodies are under reported.

\section{Case Presentation}

A 7-year-old African American patient presented to our Family Medicine practice with a chief complaint of continuous vaginal bleeding for 2 weeks. She was otherwise healthy and meeting developmental and physical milestones. She had not developed breast buds and there was no history or suspicion of sexual abuse. Her school performance was above average, and her diet was well rounded and included fruits vegetables and proteins. She had no family history of bleeding disorders.

Her physical exam revealed a healthy playful, well hydrated, well developed child in no distress. Her growth curve that put her in the $45^{\text {th }}$ percentile for weight and the $75^{\text {th }}$ percentile for height. She was cooperative with most of the exam. There were no abnormal findings on her abdominal exam. She was staged as a Tanner 1 with no pubic hair, nor secondary signs of estrogen effects or breast buds.
The vaginal exam was limited to the external genitalia due to cooperation, and revealed normal external genitalia with no lesions, no external bleeding source, and an intact hymen. There was very slow vaginal bleeding noted at the exam.

The patient was scheduled for a pelvic ultrasound and laboratory testing including coagulation studies, complete blood count, all of which were normal.

The slow vaginal bleeding persisted for several more days, and a decision was made to perform an examination under light sedation to improve cooperation and decrease emotional stress.

The examination under anesthesia included vaginoscopy and found a small amount of a foreign body within the vagina that when the material was evaluated, it was found to be toilet tissue.

The vagina was irrigated with normal saline. After the irrigation and removal of the foreign body the patient's bleeding stopped and has not returned.

\section{Summary and conclusions}

Prepubertal vaginal bleeding is an uncommon condition which requires a thorough history and GU exam for diagnosis. There are multiple causes of vaginal bleeding in the pediatric patient but there are limited literature on the etiology of foreign bodies. Often times it is also difficult to obtain a history from a pediatric patient. Common presentation is vaginal bleeding with foul smelling discharge. Pelvic ultrasound is usually the first modality of imaging for diagnosis but does not always aid in identifying foreign bodies and can be inconclusive if the object is not large enough. Therefore, if there is still high suspicion, despite a negative ultrasound, an X-ray may be obtained yet is limited to radiopaque objects [4]. Within a timely manner, if one

${ }^{\star}$ Correspondence to: Charles Haddad, Department of Community Health and Family Medicine, College of Medicine, University of Florida, Jacksonville, Florida, USA, Tel: 904-322-0665, E-mail: chaddad2@bellsouth.net

Key words: foreign body, prepubertal vaginal bleeding, sexual abuse, vaginoscopy

Received: September 02, 2019; Accepted: September 17, 2019; Published: September 23, 2019 
or more modes of imaging are negative and symptoms persist or are recurrent it is necessary to perform a vaginoscopy, which is ultimately necessary to remove a foreign object. Recent retrospective study focused on evaluating results of vaginoscopies performed on patients with vaginal discharge and noted that $20 \%$ of patients were noted to have foreign bodies [5]. In conclusion, foreign bodies as the cause of pre-pubertal vaginal bleeding is underreported and literature barely exist. Vaginoscopy is necessary for treatment regardless of imaging results but may be considered prior to exam under anesthesia [5].

\section{Conflict of interest}

There is no conflict of interest for any of the authors.

\section{References}

1. Howell J, Flowers D (2016) Prepubertal vaginal bleeding: etiology, diagnostic approach, and management. Obstet Gynecol Surv 71: 231-242 [Crossref]

2. Soderstrom HF, Carlsson A, Borjesson A, Elfving M (2016) Vaginal Bleeding in Prepubertal Girls: etiology and clinical management. J Pediatr Adolesc Gynecol 29: 280-285 [Crossref]

3. Kyrgios I, Emmanouilidou E, Theodoridis T, Galli-Tsinopolou A (2014) An unexpected cause of vaginal bleeding: the role of pelvic radiography. BMJ Case Rep 2014: bcr2013202958 [Crossref]

4. Nayak S, Witchel S, Sanfilippo J (2014) Vaginal Foreign Body: A Delayed Diagnosis J Pediatr Adolesc Gynecol 27: e127-e129 [Crossref]

5. Ekinci S, Karnak I, Tanyeli F, Ciftci A (2016) Prepubertal vaginal discharge: Vaginoscopy to rule out foreign body. Turk J Pediatr 58: 168-171 [Crossref]

Copyright: ( 2019 Haddad C. This is an open-access article distributed under the terms of the Creative Commons Attribution License, which permits unrestricted use, distribution, and reproduction in any medium, provided the original author and source are credited. 